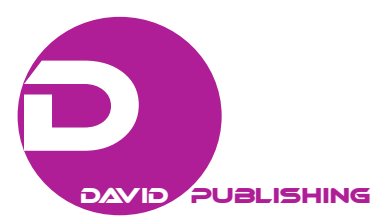

\title{
The Energy Performance in the Italian Public Administration: State of the Art and Future Prospects
}

\author{
Ornella Malandrino, Daniela Sica \\ University of Salerno, Salerno, Italy \\ Stefania Supino \\ San Raffaele University, Rome, Italy
}

\begin{abstract}
The commitment to improve energy and environmental performance in public administration is essential for the success of development models geared towards lasting sustainability. The Public Administration (PA) in Italy, in particular, plays a key role to affirm models of development oriented towards energy and environmental sustainability, thanks to a wider and more innovative vision. The PA has a dual consumer/user role, public heritage and decision maker/planner, in promoting energy efficiency at local level, in the light of specific problems and peculiarities. For several decades, initiatives have been launched at various levels to improve energy and environmental performance in the public administration. The aim of this study is to outline from a critical perspective, the state of the art of policy makers in Italy concerning energy efficiency measures in public administration. There are however many cultural, value-related, financial, technological, institutional, and operational issues in the PA sector that limit investments in energy efficiency. It should be noted that if on one hand the PA shows a lack of knowledge and is unwilling to adopt practices and systemic tools for monitoring and control, on the other, in terms of bureaucracy, the system appears to be far too complicated and costly. The multiple benefits linked to improved energy performance would therefore require that the PA rethink their organizational and functional models, put in place more flexible and less bureaucratic forms of management and a more dynamic, pervasive, and proactive approach towards initiatives that promote energy efficiency. The research is a contribution towards identifying the driving forces behind potential systems and tools for energy efficiency in the PA, highlighting both critical elements and opportunities and, in particular, the limits deriving from the technological, managerial, and organizational options available for energy efficiency enhancement in the sector of the Italian PA.
\end{abstract}

Keywords: energy efficiency, Public Administration, policy maker, sustainability development, low carbon economy, energy performance

Ornella Malandrino, associate professor, Department of Business Sciences-Management \& Innovation Systems (DISA-MIS), University of Salerno, Salerno, Italy.

Daniela Sica, research fellow, Department of Business Sciences-Management \& Innovation Systems (DISA-MIS), University of Salerno, Salerno, Italy.

Stefania Supino, associate professor, Department of Human Science and Promotion of the Quality of Life, San Raffaele University, Rome, Italy.

Correspondence concerning this article should be addressed to Daniela Sica, DISA-MIS, University of Salerno Via Giovanni Paolo II, 132, 84084 Fisciano (Salerno), Italy. 


\section{Introduction}

The commitment to the search for an improvement of the energy performance and therefore, environmental in PA represents a crucial step for the affirmation of the development models marked by a lasting sustainability.

In this scenario, the PA is called to assume a leading role in virtue of the changes that have characterized in time and space the community of reference and which have required the PA to adopt innovative ways of government, according to the logic of governance. A shared government, based on the interaction between state, market and civil society to improve the effectiveness of public policies and to meet the requirements of complexity, differentiation, and dynamism resulting from the socio-economic system (Jackson \& Stainsby, 2000).

The PA, in its various articulations, in fact, covers the dual role of consumer/user, in an optical privatistic nature, within the framework of activities related to the management of public assets and of the maker/planner, for the promotion of energy efficiency in the territory, in the light of the knowledge of the related problems and peculiarities. For these reasons, through an enlarged and innovative vision, it is being asked to define and promote policies and actions for the improvement of energy efficiency.

For several decades, a plethora of initiatives have been undertaken at various levels to improve the energy and environmental performance of the public administration, shifting paradigms from the current socio-economic development perspective, to review them from the "sustainable" development point of view.

Since the United Nations Conference on Environment and Development, held in Rio de Janeiro in 1992, useful operational tools have been introduced and used by local authorities in order to ensure effective and efficient land management, based on the fundamental concept of "accountability". Accountability is concerned not only with reporting non-financial performance, but also a set of promotional and information activities, through transparent decision-making processes (CNDCEC, 2011).

The aim of this paper is to make a critical analysis of the policies and strategies defined at national level for the improvement of the energy performance of PA in Italy. This is the awareness of the growing attention in the New Public Management toward patterns of production green and purchasing decisions based on a more sustainable socio-environmental issue.

Consequently, a brief initial analysis is carried out of the main initiatives taken at various levels to optimize energy and environment performance in the PA. Subsequently, the detecting and measurement systems currently in use that provide the data necessary to evaluate potential areas for improvement are set out and finally, the problematic nature and potential for integration of the concept of energy efficiency in management practice in the sector of the Italian PA is explored.

\section{Background}

The attention of policy makers has long focused on monitoring programs and energy demand management, defining tools, aims, and objectives.

On the thrust of the various initiatives undertaken at European level, Italy has adopted, in 2007, the Action Plan for Energy Efficiency_subsequently revised in 2011 and in 2014-and, in 2013, the National Energy Strategy (SEN).

In the first, were defined programs and measures to achieve the goal of energy savings required by Directive 2006/32/EC on end-use efficiency of energy and in energy services-recently replaced by Directive 
2012/27/EU—equal to 9.6\% per year, to be achieved by 2016 (about 11 million tons of oil equivalent—11 Mtoe/year) (Ministry of Economic Development, 2014).

The SEN, address and programming tool of the national energy policy established the priorities for the development of the energy sector (Ministry of Economic Development, 2013). Energy efficiency is the first among those identified, thanks to its ability to offer a significant contribution to the achievement of all the main energy policy objectives identified, i.e. the competitiveness, sustainability, security, and economic growth.

The measures taken at national level to achieve these ambitious targets are diversified, some already in place for some time, other in via deployment, articulated on the basis of individual areas of interest and based on a regulatory framework perhaps too wide and articulated.

However, the most important instrument used in our country for the promotion of energy efficiency, transverse and adaptable in all economic sectors (residential, tertiary, industry, and transport) is constituted by the Energy Efficiency Certificates (TEE), also known as White Certificates (CB). The CB mechanism is based on quantitative obligations to increase energy efficiency to the load of the distributors of electricity and gas, which must be achieved through the use of a market mechanism such as that of tradable certificates. The latter is exchanged between operators or on the basis of bilateral contracts, or in a market which is specially established and regulated by the manager of the energy markets (GME), through the rules laid down by the Authority for Electricity and Gas (AEEG) (Supino, Malandrino, \& Sica, 2012).

This mechanism has helped to stimulate the emergence of a market for energy services and has put in place the necessary conditions for a wider spread between economic operators and citizens of the culture of energy efficiency and the rational energy use, an essential element for a greater diffusion of more efficient technologies (De Paoli \& Bongiolatti, 2006).

The many initiatives in Italy have had effects, leading in the course of the last decade to a progressive improvement of energy efficiency in different sectors, although with significant differences in the specific contributions. Residential has in fact showed regular and steady progress since 1990, which have resulted in more than a decade, improving energy efficiency by 34\%; industry has recorded important improvements, especially in the last six years (about 7\%); finally, the fluctuating trend that has characterized the transport sector, has allowed modest and slow improvement of the overall energy efficiency, since the greatest increases achieved in certain modes of transport (plane, car, and rail) were offset by losses of efficiency of light vehicles and trucks, which are the main mode of transport of goods (Odysee-Mure, 2015).

Italy has a final energy consumption per capita among the lowest in Europe, to equality of industrial development, equal to 2.4 tep/per capita. The energy intensity, which represents the synthetic indicator more significant of the interrelationship between energy consumption and dynamics of economic development of a country-expressed by the ratio between the physical energy demand and the GDP-presents in Italy values well below the average of the 28 states of the European Union (AEEG, 2015). Obviously this result derives not only from the structural characteristics and socio-cultural traditions, but also from technological solutions made widely in place by the Italian industrial sector, first in response to crises world energy from the 70s, and in more recent times to the economic-financial crisis.

However, as already pointed out, there are still wide margins for energy efficiency in Italy, with respect to which an important role will have the Legislative Decree N. 102 of 4 July 2014, which transposed, with slight delay, Directive 2012/27/EU. It provides for the promotion of energy efficiency through a variety of measures, even prescriptive. In particular, promotes the energy auditing - to realize, compulsorily and periodically by 
2015 in large enterprises, in a structured and systematic way, by assessors objectives, competent and impartial—as a crucial tool in strategies to reduce energy consumption.

Particular emphasis also is given to the PA for the nodal role that can play in promoting energy efficiency at national level. In fact, the Legislative Decree no. 102/2014 imposes on the PA the annual preparation of a program for energy-saving real estate interventions formulated as a result of the mapping of the heritage public building related information on energy consumption. This program must be drawn up on the basis of appropriate energy diagnosis and monitored with the support of the National Agency for new technologies, energy, and sustainable economic development (Enea, 2013) and the Manager of Energy Services (GSE), in order to monitor progress. The Decree also consolidates for the PA the use of Green Public Procurement (GPP), which determines the constraints of purchase of products and services through the integration of social and environmental criteria in the procurement procedures of PA products and services. The latter is the main consumer-user in Italy, where purchases made from PA represent a significant item of expenditure amounting to approximately 17\% of GDP, compared to a share on average equal to $18 \%$ of GDP in other EU countries.

The adoption of green procurement practices in the PA gives rise not only to a process of rethinking of the procurement system, but contributes to rationalising the steps of use, disposal, and recycling of products, with positive effects in terms of environmental sustainability. Since 2003 the European Commission, within the framework of the Communication on Integrated Product Policy (Integrated Product Policy-IPP) is committed to inserting in the suggested criteria for public procurement, also to environmental criteria, besides those purely technical-economic, in order to facilitate as much as possible the diffusion of products and services that are compatible with the environment. This Communication is part of a broader Action Plan on Sustainable Consumption and Production and Sustainable Industrial Policy-SCP/SIP, which provides a set of integrated tools to be taken to implement the paths to improve the energy performance and environmental characteristics of products and services used and/or provided by public bodies (Enea, 2013).

In Italy the process of adoption of the Green Public Procurement policy has been quite gradual, scanned in time from the issuing of several provisions which impose on Public Administrations the purchase of "green" products. In 2006, was issued the Legislative Decree no. 163, while, in 2008 with the Ministerial Decree of 11 April 2008 (Official Gazette no. 107 of 8 May 2008), was enacted on the Action Plan for sustainable consumption in the Public Administration (PAN GPP), recently updated with the Ministerial Decree of 10 April 2013.

The latter provides for the definition of "Minimum Environmental Criteria" (CAM), for certain "product categories", which represent the point of reference at national level in the field of green public procurement.

These criteria have the purpose to support public administrations in the realization of the purchasing procedures that minimise the environmental impacts of products/services along the entire life cycle, i.e. in a Life Cycle Assessment (LCA) perspective.

\section{Constraint and Opportunities for Improving Energy Efficiency in the Public Administration in Italy}

Since the 1990s, the Italian organism public administration, consuming more than 1,000 tons, has been obliged to appoint an Energy Manager (EM) to promote and spread a culture of energy efficiency within the organization. 
However, in spite of regulatory constraints, to date the Italian PA has appointed very few Energy Managers and remains distinguished by much inefficiency.

In 2015, 2,100 Energy Managers were appointed, of whom only 150 within the PA. While it is impossible for us to make a more accurate estimate, the regulation appears to have been widely disregarded, since eight to nine out of 10 public bodies have failed to comply; only three Energy Managers are employed by ministries and agencies, only seven out of 20 regions and 43 out of 110 provinces have appointed an Energy Manager, and local authorities have only 105 instead of one thousand (Fire, 2013).

Therefore, it is a well-known fact that the PA's commitment to improve energy performance is long overdue even if recent studies have estimated that by 2020 the PA could save electricity and thermal energy equal to 0.8 TWh and 1.5 TWh respectively through intervention capable of generating substantial investments valued at approximately $€ 1,000$ billion per year (Politecnico di Milano, 2013).

The driving forces for implementing systems and tools to improve energy efficiency range from economic and organizational oriented incentives to those related to environmental and social dimensions. The high cost of energy makes it essential to identify rapidly the most appropriate technological and organizational interventions for reducing the energy costs of the PA and its exposure to volatile energy prices.

In order to improve energy performance, the energy management practices of the PA must be in synchrony with the concept of energy efficiency, effective detection systems have to be implemented and suitable data and information must be available for evaluating the results obtained in order to identify possible areas of improvement.

There are however many cultural, value-related, financial, technological, institutional, and operational issues in the public administration sector that could limit investments in energy efficiency, as shown in Figure 1. It is particularly important to note that if on one hand the PA shows a lack of knowledge and unwillingness to adopt practices and systemic tools for monitoring and control, on the other, it appears to be too complicated and costly. In fact, specific skills and high technical and organizational capabilities are required when implementing programs aimed at efficient and sustainable energy management.

There are also other problems such as the persisting limited availability of financial resources, which discourages the PA from making plans and investments that could yield returns in the medium-long term, that call for the realization of effective energy efficiency improvement strategies.

In addition, the lack of awareness of the benefits and opportunities related to the improvement of energy performance does not permit the development of a "culture" of shared energy efficiency.

Culture in this case expressed both in "socio-environmental" terms (e.g., environmental protection, enhancement of energy, and renewable resources, etc.) and in "individualistic" terms (e.g., everyday use energy conscious, lifestyle, buying patterns, etc.) depends on the degree of sharing of objectives, strategies, and values that the PA can spread internally and among the various external stakeholders. Internally, through the virtuous cycle of strategic sharing and co-responsible design, using communication and training to "contaminate" vertically and across the organization and stimulate feedback flows; externally, through the enhancement in a communicational key, of what has been achieved and the involvement of all stakeholders, whatever their title of relationship with the organization.

From a technical and operational perspective, the complexity of the planning, technical, and financial aspects of energy efficiency measures, as well as the lack of commitment, also relational, of the individuals responsible for their implementation, magnifies and reinforces the barriers to energy efficiency. 
Additionally legislative instability and the long and tortuous bureaucratic procedures required by global operations are factors that induce the PA to opt for simple technologies to implement rapidly but characterized by short-term vision.

One way of overcoming knowledge barriers and reducing technical and economic information asymmetries, may be to enforce the regulations currently in force.

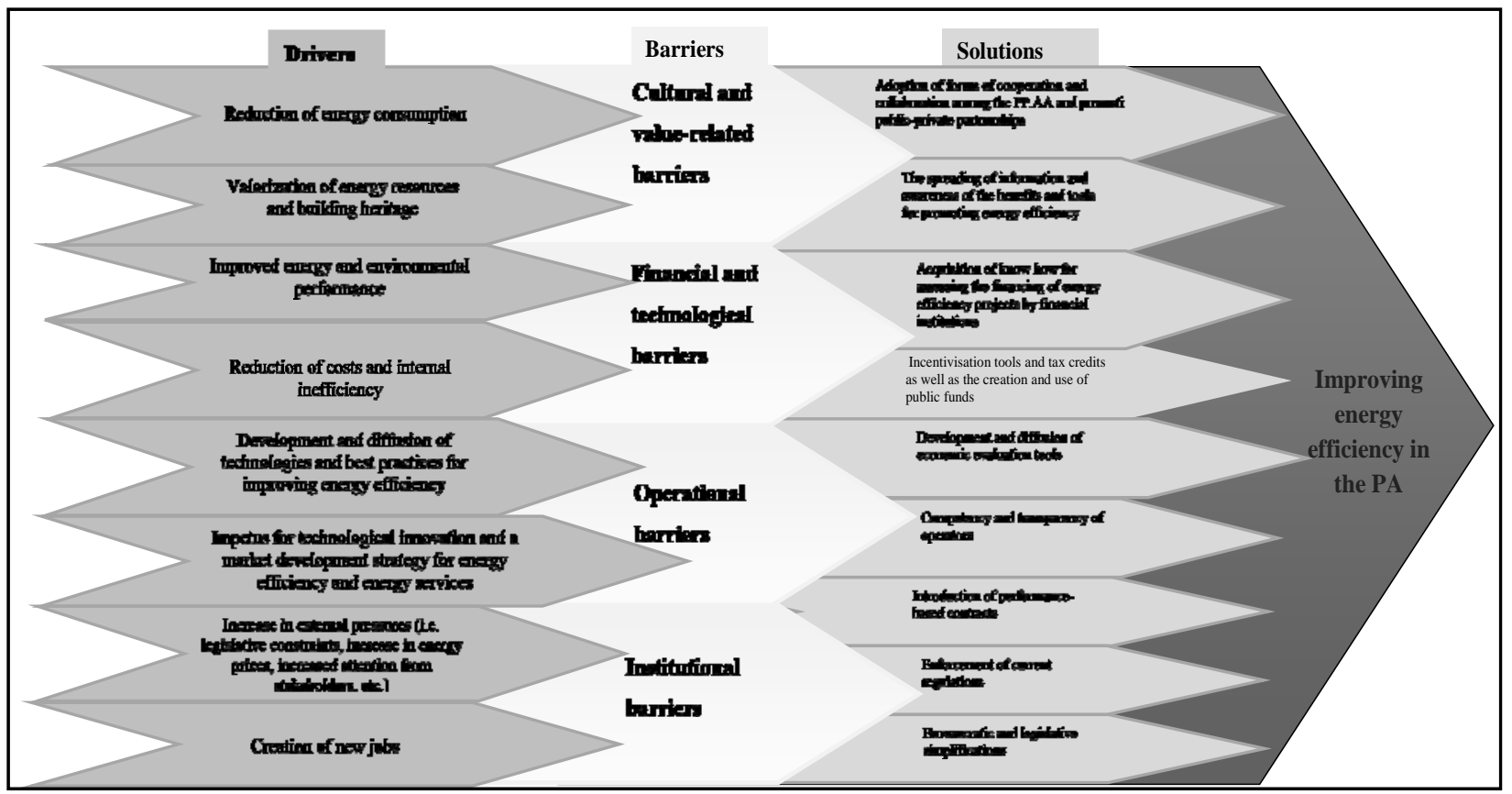

Figure 1. Streamlining energy efficiency in the PA.

\section{Conclusion}

The affirmation of energy paradigms geared towards greater efficiency and sustainability is an interesting challenge and presents new opportunities for the Italian PA, which should play a leading role in the complex economic scenario that is emerging for some years.

The current energy efficiency approach of the Italian PA stems from a strategically unstructured vision, governed by legislation and highly fragmented planning that result in occasional activities rather than medium-long term planned initiatives, enacted after a careful measurement and assessment of the results obtained.

Therefore, it appears that the boundaries of the PA require redefining in order to reconsider the relationships between the public and private sectors and the public institutions in terms of cooperation, collaboration, and partnership, based on reliable and long-lasting relationships, integration of public funds and private resources and the resultant risk allocation. Moreover, it is essential to exchange experiences and best practices with the aim of guiding the PA and especially local bodies towards lasting sustainability and economic growth through competitiveness, environmental protection, and social development.

\section{References}

Autorità per l'Energia Elettrica e il Gas. (2015). Relazione annuale sullo stato dei servizi e sull'attività svolta. Available from http://www.autorita.energia.it [Accessed 21/12/2015] 
Consiglio Nazionale dei Dottori Commercialisti e degli Esperti Contabili-Cndcec. (2011). Contabilità e rendicontazione di sostenibilità del sistema della pubblica amministrazione e degli enti locali. Policy Statement. Available from http://www.cndcec.it/Portal/Default.aspx [Accessed 12/02/2016]

De Paoli, L., \& Bongiolatti, L. (2006). The promotion of energy efficiency in Italy. Economics and Policy of Energy and the Environment, 3, 29-68.

Enea. (2013). Rapporto annuale efficienza energetica 2012. Available from http://www.enea.it/it/produzione-scientifica/pdf-volumi/VRAEE_2012.pdf [Accessed 21/10/2015]

FIRE. (2013). Rapporto sugli Energy Manager in Italia: Evoluzione del ruolo e statistiche. Available from http://www.fire-italia.it/ [Accessed 21/01/2016]

Jackson, P. M., \& Stainsby, L. (2000). Managing public sector networked organizations. Public Money \& Management, $20,1$.

Ministero dello Sviluppo Economico. (2014). Piano d'Azione Italiano per l'Efficienza Energetica. Available from http://www.sviluppoeconomico.gov.it [Accessed 12/11/2015]

Ministero dello Sviluppo Economico. (2013). Strategia Energetica Nazionale: Per un'energia più competitiva e sostenibile. Available from http://www.sviluppoeconomico.gov.it [Accessed 9/11/2015]

Odysee-Mure. (2015). Profilo di Efficienza Energetica: Italia. Available from http://www.odyssee-mure.eu/publications/profiles/italy-efficiency-trends-italien.pdf [Accessed 12/02/2016]

Politecnico di Milano. (2013). Energy efficiency report. L'efficienza energetica in Italia: soluzioni tecnologiche ed opportunità di business nell'industria, i servizi e la Pubblica Amministrazione. Available from http://www.energystrategy.it/ [Accessed 05/12/2015]

Supino, S., Malandrino, O., \& Sica, D. (2012). Il contributo dei certificati bianchi al miglioramento dell'efficienza energetica in Italia. Esperienze d'impresa, 1, 93-105. 